Veritas日 Scientia

Vol. 8. $\mathrm{N}^{\circ} 1$.

Enero - Junio del 2019

ISSN Edición Online: 2617-0639

https://doi.org/10.47796/ves.v8i1.117

\title{
MODELO DE VERACIDAD DE ENCUESTA BASADA EN UNA APLICACIÓN ANDROID PARA ZONAS URBANAS Y RURALES
}

\author{
VERACITY MODEL OF SURVEY BASED ON AN ANDROID APPLICATION FOR URBAN AND RURAL \\ ZONES
}

Álvaro Oswaldo Luque Fernández ${ }^{1}$

Presentado: 08/04/2019

Aceptado: $10 / 06 / 2019$

Publicado online:17/07/2019

\begin{abstract}
RESUMEN
Las empresas consultoras que se dedican a la investigación de mercado y la elaboración de proyectos públicos y privados, vienen enfrentándose al tiempo excesivo que incurren en el trabajo de campo, lo cual trae como consecuencia la pérdida de clientes y bajo compromiso de los servicios que se ofrecen. Durante la toma de datos, se encuentra un error no muestral, que proviene del entrevistado, donde no es lo suficientemente objetivo y sincero con el diligenciamiento de la encuesta. Para este estudio se considera los patrones de veracidad para admitir una encuesta que puede provenir de: cuestionarios, escalas o inventarios. El objetivo de este estudio, fue desarrollar una aplicación android que permita elaborar un modelo de veracidad de encuestas en zonas urbanas y rurales. Bajo este contexto, la investigación muestra una solución basada en un modelo de veracidad de encuestas, que se basa en una aplicación android y que puede ser utilizada en cualquier distrito, también permite generar supervisiones al personal (encuestadores) que realizan trabajo de campo y finalmente realizar el seguimiento de las cuotas bajo parámetros poblacionales. La presente investigación, de acuerdo a las características del problema es de tipo aplicada de nivel relacional y diseño experimental, la población objeto de estudio se concentró en los CPM de Ancocala y Calacala del distrito de Cairani de la provincia de Candarave, en una muestra constituida por 96 hogares, siendo el informante el jefe de hogar, por lo tanto, la muestra es no probabilística y por conveniencia, dado que solamente servirá para comprobar el grado de veracidad de la encuesta. Se logró aplicar patrones programables en una aplicación android para generar un modelo de veracidad de encuestas $(87.50 \%$ de veracidad y $12.50 \%$ de mentiras en las encuestas aplicadas). Se concluye que el método tradicional no lograba detectar la sinceridad en cuanto al diligenciamiento de las encuestas basadas en los niveles socioeconómicos.
\end{abstract}

\footnotetext{
${ }^{1}$ Comunicador y Maestro en docencia universitaria y gestión educativa.

2 Docente universitario (Facultad de Ingeniería - Universidad Privada de Tacna), experto en estadística para la investigación y en gestión de proyectos públicos y privados. Coordinador de Investigación de la Escuela de Postgrado de la UPT
} 
Palabras clave: smartphone, android, encuestas, modelo de veracidad, geolocalización.

\begin{abstract}
The consulting companies that are engaged in market research and the development of public and private projects are facing the excessive time spent in the field, which results in the loss of customers and under the commitment of the services that are offered. During the data collection, there is a non-sample error, which comes from the interviewee, where they are not sufficiently objective and sincere with their performance on the survey. For this study we consider the veracity patterns to admit a survey that can come from: questionnaires, scales or inventories. The objective of this study was to develop an android application that allows to elaborate a veracity model of surveys in urban and rural areas. In this context, the research shows a solution based on a model of veracity of surveys, which is based on an android application and that can be used in any District, it also allows to perform supervisions on the personnel (pollsters) that perform field work and finally to monitor the assessment under population parameters. The present research, according to the characteristics of the problem is of an applied type of relational level and experimental design, the population study was concentrated in the Ancocala and Calacala CPMs of the Cairani district of the province of Candarave, with a sample of 96 households, the informant being the head of household, therefore the sample is non-probabilistic and for convenience, since it will only serve to verify the degree of veracity of the survey. It was possible to apply programmable patterns in an android application to generate a model of veracity of surveys $(87.50 \%$ of truthfulness and $12.50 \%$ of lies in the applied surveys). It is concluded that the traditional method failed to detect the sincerity in the performance of the surveys based on the socioeconomic levels.
\end{abstract}

Keywords: smartphone, android, surveys, accuracy model, geolocation

\title{
INTRODUCCIÓN
}

Hoy en día, muchas personas no se imaginan estar un solo día sin su dispositivo móvil. Los usuarios actualmente encuentran información rápidamente a través de buscadores, constantemente se mantiene conectado con amigos y familiares. A menudo, se suele llegar al punto, en que se desea tener una aplicación para situaciones laborales, académicas o particulares, para ciertas necesidades, sin embargo, estas aplicaciones no se encuentran en internet. El desarrollo de una aplicación suele llevar mucho tiempo y necesita profesionalismo en el desarrollo de software o aplicaciones a medida; y como los usuarios o empresas no encuentran estas aplicaciones, tienden a esperar a que alguien la desarrolle, o tenga que dirigirse a la web y pedirle a la gente poner en práctica sus ideas.

Los dispositivos móviles son utilizados para diversos fines, como la navegación móvil a través del google, juegos, envío de mensajería electrónica como el gmail, usos de redes sociales, sistema de geolocalización, ingreso a Youtube, instalación de aplicaciones (APPs) bancarias para la realización de transacciones bancarias, capacitaciones virtuales, entre otras, la tendencia es positiva antes la posesión de un teléfono móvil multimedia. En la India, Patil, Sawant, Desai \& Shinde (2018), desarrollaron una aplicación basada en GPS, para alertar a los usuarios a través de una alarma, esta aplicación para Android TASK TRIGGER ayuda al usuario a recordar las tareas que debe hacer en la 
ubicación específica. En Singapure, Cottrill, Cámara, Zhao, Ferreira, Beng, Ben-Akiva, y Zegras (2013) elaboraron una encuesta de movilidad futura para viajes, basada en teléfonos inteligentes;

El sistema de posicionamiento (GPS) ha evolucionado en cuanto a precisión y ubicación como los usuarios requieren. Sin embargo, la ubicación de la red de Android requiere de un proveedor de telefonía móvil para utilizarlas en las señales de Wi-Fi, tanto dentro y fuera de edificaciones. En la actualidad, alrededor de 1,300 fabricantes de teléfonos en el mundo utilizan Android en sus smartphones. Existen en el mercado más de 24 mil modelos de todas las gamas, colores y precios, los cuales hacen que Google tenga más de 2 millones de móviles activos. En la India, Kushwaha \& Kushwaha (2011), generaron servicios basados en localización mediante el uso del sistema operativo android, la investigación presentó una serie de aplicaciones diferentes donde proporciona información al usuario con respecto a un lugar que desee visitar, dado que estas aplicaciones no deberían de estar limitadas a escritorios solamente, sino que dichas aplicaciones deben de importarse a los diferentes dispositivos móviles, de esta manera una persona no necesita llevar al viaje guías cuando visita otros lugares.

Según el Instituto Nacional de Estadística e Informática (INEI) 2018, el 92.4\% de los usuarios de Internet que se encuentran entre los 25 a 40 años de edad acceden a través de sus teléfonos móviles, cifra que representa un incremento de 9.4 puntos porcentuales, en comparación con el trimestre abril-mayo-junio de 2017. En el Perú, las empresas consultoras que se dedican a la investigación de mercado y la elaboración de proyectos públicos y privados, viene enfrentándose al tiempo excesivo que incurren en el trabajo de campo, en sus diferentes etapas: distribución de cargas de trabajo, supervisión a encuestadores, digitación de encuestas; en consecuencia la presentación de los hallazgos y elaboración del informe, traen como consecuencia pérdida de futuros clientes y bajo compromiso de los servicios que se ofrecen.

En Lima, Ku Lam (2013), implementó una plataforma interactiva móvil orientada al aprendizaje en temas de física, la investigación se realizó tomando como plataforma el diseño y la implementación de un aplicativo celular en Android cuya intención fue el de promover a los alumnos en la fase escolar, edad en la que los jóvenes pierden corridamente el beneficio por los temas académicos. Gáloc (2016), diseñó e implementó un sistema de geolocalización en interiores para plataforma Android vía la red Enterprise WLAN de la Pontificia Universidad Católica del Perú; a su vez Cornejo (2013), implementó una herramienta que permitió coadyuvar a tener una mejor gestión del proceso de incidencias, abarcando uno de los procesos necesarios para validar la certificación de ISO 20000 en una empresa dedicada al sector Tecnología e Información. Estos últimos años se han incrementado aplicaciones en el Perú. Urbano (2015), construyó un sistema de creación y aplicación de encuestas que soporte preguntas tipo matriz de menús desplegables, basado también en un sistema de encuestas.

Esta investigación ayuda a resolver el problema que existe actualmente con la veracidad durante el recojo de datos en una encuesta; también se realiza seguimiento al encuestador mediante geolocalización y control en su carga asignada, de esta manera se conocerá el lugar donde se encuentre haciendo el trabajo de campo; también se minimizarán los errores en la inconsistencia de los datos a través de patrones y reglas de validación; se tendrá información en tiempo real para monitorear el cumplimiento de las cuotas asignadas elevando la representatividad de la población objeto de estudio; como consecuencia se minimizarán los índices de inconsistencia y eliminarán el proceso de digitación en planta. La recolección de datos a través del uso del tablet (dispositivos móviles personales) es más rápida que la recolección de datos mediante métodos tradicionales y puede ser todavía más rápida apenas se tenga acceso a internet, mediante controles se incrementan los índices de veracidad, de tal forma que se minimizarán las inconsistencias durante la toma de datos. La presente investigación es importante porque resuelve un problema latente en las empresas de investigación, dado que elevaría los índices de veracidad, reduciría los errores e inconsistencia de datos; de esta manera se eleva la satisfacción de la entidad contratante o población gracias al método propuesto. 
La aplicación móvil tiene amplios usos de funcionamiento, como: llamadas, mensajería, navegación, chat, redes sociales, comunicación, audio, video, juego etc. En gran número de aplicaciones móviles algunas están preinstaladas en el móvil y otras pueden descargarse desde internet. Desde el punto de vista técnico las diferentes aplicaciones móviles son ejecutar capaz en diferentes plataformas gestionadas como iPhone, BlackBerry, Android, Symbian, Windows (INFODASA, 2015). También ha influido en este crecimiento la llegada al mercado de las "tabletas", dispositivos móviles con una pantalla de mayores dimensiones y donde también pueden consumirse aplicaciones con algunas funcionalidades potenciadas. (Mobile Marketing Association, 2011).

Las aplicaciones empresariales, también conocidas como aplicaciones de productividad, ocupan el segundo lugar con el considerable 10\% de participación. Los teléfonos inteligentes de hoy en día son capaces de realizar muchas tareas complejas en la ejecución. Facturar, comprar, reservar, enviar correos electrónicos, rastrear el progreso del trabajo, lo que sea. Las aplicaciones empresariales varían de aplicaciones B2B a aplicaciones de oficina y personales para aumentar la productividad y minimizar los gastos: imagine que puede encontrar desarrolladores de aplicaciones de Android para contratar, contratar un nuevo gerente de ventas o comprar nuevos cartuchos para las impresoras de su oficina con solo un toque.

La Encuesta, constituye uno de los medios más importantes y oportunos para la obtención de información estadística, social, demográfica y económica, proveniente de los encuestados o informantes, que permitirá medir indicadores socio demográficos de la población, el análisis y diseño de políticas en el área social y la evaluación del impacto de las mismas en las condiciones de vida de la población, etc.

Cuando se hace uso del método Directo, es decir, el encuestador visitará al público objetivo en sus puntos de ubicación (viviendas de cada distrito u otros), tomará la información de parte del encuestado y completará los formularios que se requieran aplicar. Los períodos de referencia de la encuesta son variados dependiendo del tema a tratar y de la ubicación de los encuestados.

La elección de un aparato electrónico para recoger datos durante el trabajo de campo, es de suma importancia, dado que el personal que realiza las encuestas se moviliza de una manera dinámica en contraste a aquello que no se le da importancia y sin embargo se debería de tomar en cuenta como factor crítico en la implantación del sistema. De hecho, el tratamiento de la información ha generado que tengamos a nuestro al alcance diversos tipos de dispositivos, en concreto las tablets, adecuadas en su mayoría para utilizar en el hogar o dentro de las organizaciones. (PNUD, 2015).

La recolección de datos es la actividad que empieza una vez definido el objetivo y el diseño de la investigación, y acaba cuando se dispone de los datos recogidos para proceder a su análisis (Ochoa, 2015). En este contexto, "efectivo» significa encontrar el equilibrio entre costos, tiempo y calidad. Las metodologías convencionales para la recolección de datos, como: las entrevistas personales y por teléfono no son tan seguras respecto a la veracidad al momento de la captura de datos y por lo tanto las consultoras se ven obligadas a investigar y aplicar nuevas tecnologías para incrementar los índices de veracidad. El encuestador, es un actor importante en la investigación estadística, encargado de entrevistar a la unidad de análisis y acopiar información sincera por parte de los entrevistados. Se debe desempeñar con responsabilidad, compromiso y profesionalismo, requisitos indispensables para obtener información útil y de calidad (INEI, 2014). Las nuevas tecnologías han creado un nuevo comportamiento al realizar un diagnóstico, o un estudio de mercado, por lo que la interacción entre el encuestador y encuestado ha cambiado (García, 2012). Las nuevas tecnologías han dado lugar a nuevas formas de recolectar información sobre los encuestados. Desde el auge de Internet a mediados de los años '90, la investigación se ha volcado en la recolección de datos online. Para la aplicación de encuestas online/offline y recolección de datos, se propone el uso de iPads, y dispositivos Android para recoger datos en cualquier lugar, por lo que se diría adiós al papel, a los formularios web y a la necesidad de conectarse a internet. Este proyecto tecnológico usa herramientas de dispositivos móviles, aporta en la reducción del tiempo en el procesamiento y como consecuencia incrementa la calidad y optimiza la difusión de los hallazgos, teniendo en cuenta el 
avance de la tecnología móvil. También, puede efectuar supervisión durante el trabajo de campo, mediante el uso del Sistema de Posicionamiento Global (GPS), direccionándose más hacia la supervisión de los encuestadores, reflejándose en la calidad de toma de datos (Albrecht, 2007). La elaboración de una aplicación bajo una plataforma móvil para encuestas y recolección de datos, permitiría crear encuestas y juntar respuestas utilizando tabletas con o sin conexión a Internet. Además, esta aplicación es útil para estudios de campo en la parte rural que por lo general no se cuenta con internet, feedback de clientes, ferias y eventos para la captación de clientes, auditorías, inspecciones, evaluaciones y cualquier tipo de recopilación de datos.

Por otro lado, se propone una plataforma para entrevistas de pantalla única, donde las entrevistas o encuestas administradas se realizarían por el mismo encuestador y las preguntas se visualizarían en una pantalla a la vez, con respuestas verificables antes de ser enviadas, bajo estándares de control; se registraría auditorías y puntaje para determinar los resultados, como también conversión de formulario de papel a formato digital en minutos.

Bajo este análisis, durante la toma de datos, se ha encontrado que existe un error no muestral que no es producto de la capacitación, sino más bien por parte del entrevistado, donde no es sincero y honesto con el diligenciamiento de encuesta, para este estudio se han considerado los patrones de veracidad para admitir una encuesta ya sea como: cuestionarios, escalas o inventarios. Adicionalmente, se cuenta con funciones interactivas capaces de capturar toma fotográfica del entrevistado o fotografías del entorno donde se realiza la entrevista. Adicionalmente se puede integrar una geolocalización (GPS) para que los encuestadores muestren una locación para determinar la ruta que se le ha asignado como encuestador rural (de dónde han venido o hacia dónde van) en un mapa digital. Se le agregarán preguntas de control para validar la encuesta.

\section{METODOLOGÍA}

La presente investigación, de acuerdo a las características del problema es de tipo aplicado, bajo un nivel de investigación será descriptivo, relacional ya que se va a trabajar con 2 variables y la relación que tienen. Para este estudió la población objeto de estudio se encontró concentrado los CPM de Ancocala y Calacala del distrito de Cairani de la provincia de Tacna; La muestra está constituida por 96 hogares, siendo el informante el jefe de hogar, por lo tanto, la muestra es no probabilística y por conveniencia, dado que solamente sirvió para comprobar el grado de veracidad de la encuesta.

La aplicación es cuasi experimental, en dos tomas de datos antes (método tradicional) y después (usando dispositivo móvil). Para el procesamiento de datos, se empleó el software estadístico SPSS. Para el análisis de datos se aplicó la prueba para muestras relacionadas. Se recolectaron datos a través de una encuesta en físico y digital, se almacenaron datos en MY SQL server, para el análisis se realizará en Rational Rose y la presentación de los resultados en tablas y figuras estadísticas.

La propuesta contiene los procesos a tener en cuenta para la mejorar la gestión en los proyectos que abordará esta investigación son antes y después de la propuesta.

\section{Respecto a distribución de carga de cuestionarios}

Antes de la propuesta, los supervisores coordinan tareas previas a la salida al trabajo de campo, como abastecimiento oportuno y suficiente de encuestas, carga de trabajo, documentos auxiliares como reportes, cuestionarios, material de oficina y otros. Abastecer oportunamente de material (encuestas) a su equipo para las encuestas. Después de la propuesta, los supervisores locales coordinan tareas previas a la salida al trabajo de campo y distribuyen a los encuestadores cargas de trabajo y tablet's asignada por segmento, previa coordinación con la Oficina de Tecnología de la Información.

\section{Respecto a la revisión los cuestionarios después de aplicar la encuesta.}

Antes de la propuesta, los supervisores deben revisar los cuestionarios después de ser aplicados por el encuestador al $50 \%$ más uno en campo y al $100 \%$ en gabinete. 
Después de la propuesta, los supervisores deben observar cuestionarios offline (de ser necesario), para levantar cualquier error u omisión en el diligenciamiento.

\section{Supervisar el avance diario de cada encuestador}

Antes de la propuesta, recepcionar la información del avance diario del encuestador y reporta itinerario en la hoja de ruta del periodo correspondiente. Embalaje inicial de cuestionarios completos. Después de la propuesta, revisar la data almacenada en la memoria del tablet y realizar una supervisión post - campo al paso del encuestador mediante tablet asignado.

\section{Diligenciamiento y digitación del cuestionario}

Antes de la propuesta, el encuestador diligencia el cuestionario a la población objeto de estudio y el digitador recepciona el material debidamente diligenciado e ingresa los cuestionarios entregados de acuerdo al cronograma.

Después de la propuesta, el encuestador diligencia diariamente las encuestas offline de acuerdo al cronograma.

\section{Generación de reporte de avance diario por parte del encuestador}

Antes de la propuesta, el encuestador entrega diariamente las encuestas aplicadas y reporta el avance diario del trabajo de encuestas al supervisor local. Después de la propuesta, generar el reporte de avance diario del encuestador y velar por el cuidado del equipamiento tecnológico tablet, guardar confidencialidad sobre la información de las encuestas offline.

\section{Control de documentos por parte de la empresa}

Antes de la propuesta, el encargado de la Unidad de Distribución y Recepción de Archivos (UDRA) y llevar el control de los cuestionarios distribuidos, así como los documentos auxiliares utilizados, durante la ejecución de la encuesta. Después de la propuesta, el encargado de Tecnología de la Información (T.I.), llevará el control de las encuestas, así como los documentos auxiliares utilizados, durante la ejecución de la encuesta.

\section{Suministro de los recursos a los encuestadores y supervisores}

Antes de la propuesta, el responsable de integración de la información suministra oportunamente a los Supervisores el material necesario para el cumplimiento de cada una de las fases de la encuesta. Después de la propuesta, el encargado de TI, suministra oportunamente a los Supervisores Locales los equipos informáticos necesario para el cumplimiento de cada una de las fases de la encuesta.

\section{Recepción de documentos físicos y respaldo de la base de datos}

Antes de la propuesta, el responsable de resguardar la información recepciona el material utilizado, de tal manera de garantizar su conservación. El encuestador vela por el cuidado del material recepcionado durante la etapa de digitación y guarda confidencialidad sobre la información de los cuestionarios. Después de la propuesta, el encargado de $\mathrm{TI}$, resguarda la data, de tal manera que garantiza su conservación.

\section{Análisis Comparativo}

Como podemos ver en ambos casos se promueve la solución hacia la digitalización de una encuesta incrementando el grado de certeza al momento del recojo de datos llamándolo para este estudio un modelo de patrones de veracidad. Podríamos decir, que antes de su implementación de la APP no se incluyó ningún plan de mejora constante para el levantamiento de encuestas, dado que se utilizaba la metodología tradicional basada en papel y lápiz. La propuesta de esta nueva metodología, busca brindar además de una correcta estructuración de la información y almacenamiento de las bases de datos en la nube, para poder posteriormente administrarla en pro de los objetivos de la Consultora.

La aplicación basada en tecnología android brinda datos más precisos y rápidos para tratamiento a través de la solución móvil propuesta, además se hace uso de un sistema de geolocalización para la 
supervisión de los encuestadores durante la toma de datos, el SIG manteniendo actualizada no solo la información de los encuestado sino también la información geo espacial.

\section{Diseño de la interfaz}

Se procedió a diseñar las principales pantallas, las cuales fueron desarrolladas con Android Studio 2.3, el mismo que nos permite visualizar en pantallas múltiples la información que se necesite. Para salir del sistema y ventanas se presentan al mismo tiempo diferente conjunto de salida. Los usuarios pueden ejecutar varios programas al mismo tiempo y presentar la salida de cada programa en una ventana. A continuación, se mostrarán algunas de las pantallas:

\section{Ingreso al sistema de entrevistas}

Para ingresar al Sistema de Encuesta, deberá colocar su usuario y contraseña con el estado de activo para su cuenta de lo contrario no le permitirá acceso. Luego "picará" en la opción INICIO.

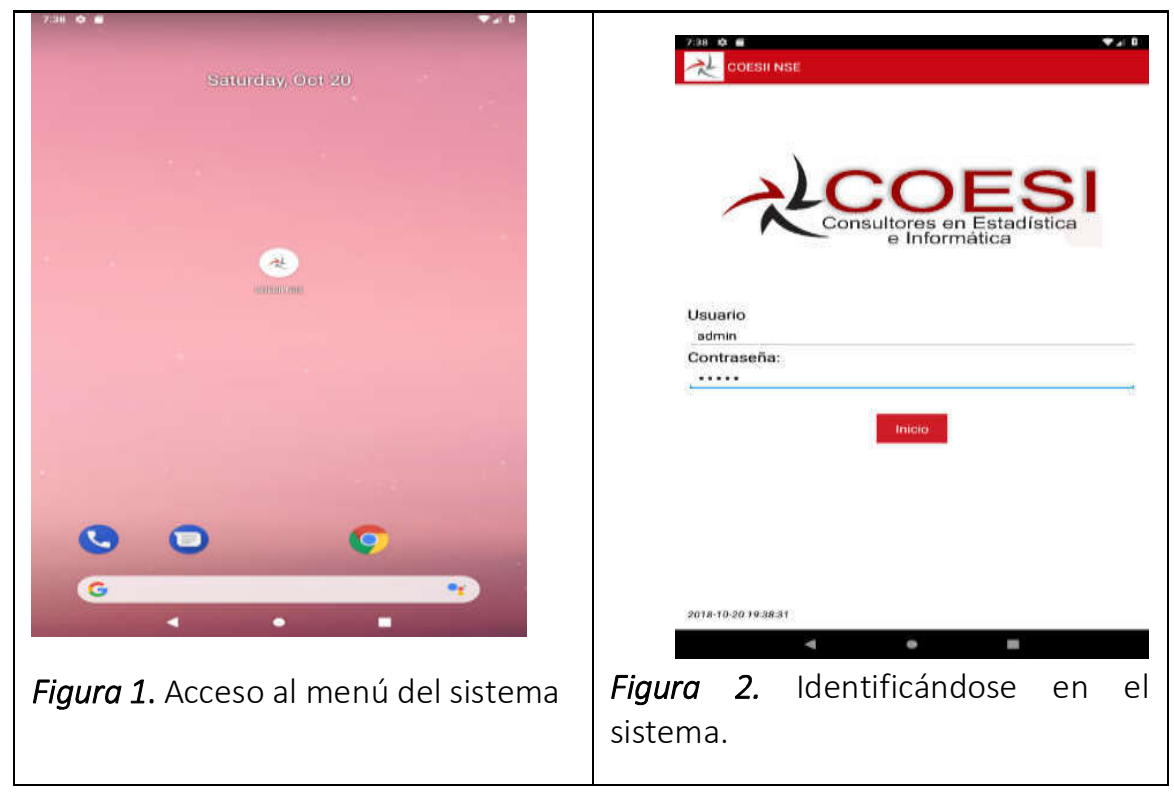

Registro de nueva encuesta

Aparece el menú principal del sistema de entrevistas

Tabla 1

Registro de la nueva encuesta

\begin{tabular}{ll}
\hline \multicolumn{1}{c}{ Opciones } & \multicolumn{1}{c}{ Descripción } \\
\hline Apertura y visitas & $\begin{array}{l}\text { Permite el ingreso a la apertura de viviendas, el registro de los hogares y } \\
\text { de las visitas efectuadas. }\end{array}$ \\
\hline Sección $1 . . .5$ & Permite el ingreso de datos por secciones. \\
\hline Cobertura & Verificación de la carga de trabajo concluida o pendiente. \\
\hline
\end{tabular}

\section{Menú utilitario}

El menú Utilitarios proporciona las opciones que permiten la salvaguarda, transferencia y mantenimiento de las bases de datos, así como el cargado de la muestra y el cargado de las viviendas de reemplazo. En la figura 4 se representa el menú de Utilitarios. Para acceder a él, desde el Menú principal hay que ingresar a la opción Utilitarios. 
Exportar a la Nube

Reparar Base de datos

Eliminar Encuestas Almacenadas

Figura 4. Menú utilitario.

Exportar a la Nube: En esta opción enviamos la información de las encuestas a nuestro servidor en la nube.

Repara Base de Datos: Permite reparar la base de datos cuando por algún imprevisto la base de datos se daña.

Eliminar Encuestas Almacenadas: Elimina todas las encuestas dentro del dispositivo.

Al seleccionar la opción de exportar a la nube, comenzará a enviar la información al servidor en la nube.

\section{Cargando la encuesta}

Al inicio de cada mes se debe cargar en la Tablet la muestra de viviendas a trabajar. Para efectuar el cargado de la muestra, desde el menú principal, debe ingresar al Menú de Utilitarios y desde allí "picar" en el botón "Nueva Encuesta". Después de lo cual, el programa presentará la pantalla de cargado.

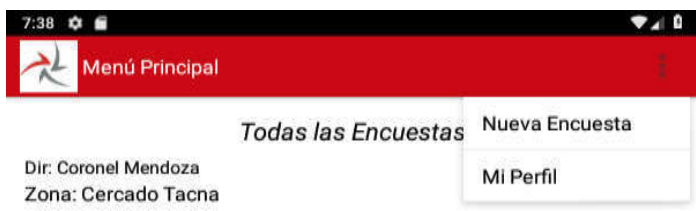

Figura 5. Inicio del cargado de encuesta.

\section{Apertura de encuesta}

El primer paso antes de efectuar las entrevistas, es la apertura de la vivienda (Figura 5). Los pasos a seguir para realizar la apertura de las entrevistas son el tipo de vivienda:

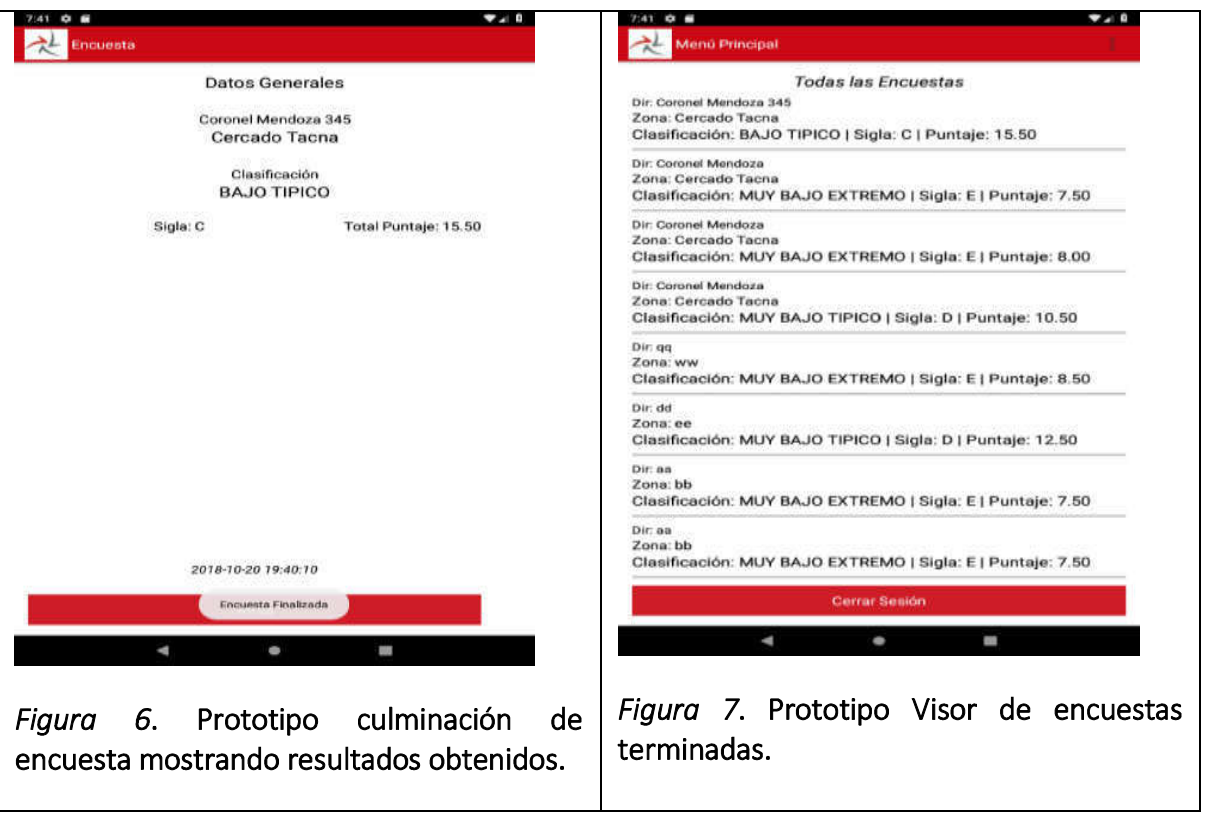




\section{Diseño de reportes}

Reportes diseñados con Html5 y Css3 para el formato y conversión a PDF y Excel basado en librerías libres de PHP. Después de distintas interacciones con el usuario, se logró diseñar los reportes requeridos. Bajando con el cursor podemos ver todas las preguntas (incluyendo las de validación), las respuestas obtenidas por el encuestado y el puntaje obtenido en dicha pregunta.

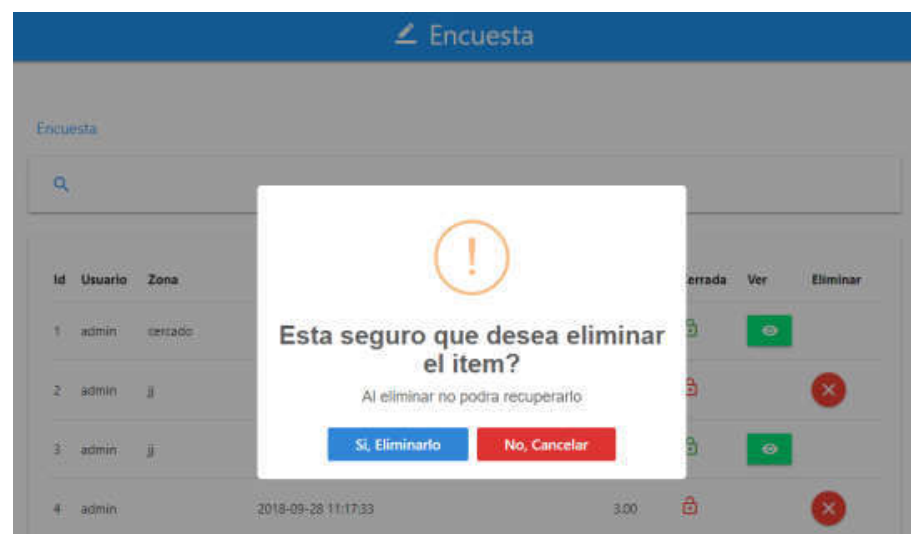

Figura 8. Prototipo Visualizar reporte de encuestas para eliminarlas.

Al presionar el aspa manda la alerta antes de eliminar las encuestas no culminadas correctamente por el encuestador.

\section{RESULTADOS}

Este estudio se realizó en la zona sur del Perú, siendo más precisos en la cuidad de Tacna entre los años 2017 y 2018, desarrollando una aplicación Android para encontrar un modelo de veracidad a través de una encuesta que mide los niveles socioeconómicos.

La información fue consolidada y actualmente se encuentra disponible en la nube (conectividad online u offline) pudiendo generar reportes personalizados en tiempo real, y de esta manera garantizar los informes actualizados y avances de los trabajo de campo; la bondad de este aplicativo fue ampliar la capacidad de compartir registros y reportes de manera interna y externa; como también automatizar la operación de campo permitiendo activar alertas, correos electrónicos, tareas, notificaciones, nuevos formularios o asignar acciones a grupos o individuos sin necesidad de intervención humana; se generarán también nuevos formularios de forma automática a partir de las respuestas capturadas; enviando mensajes internos (a través de push notifications) a los usuarios de campo a través de la APP e implementará la supervisión bajo lineamientos del uso del Sistema de Posicionamiento Global - GPS. Una vez culminada la aplicación se tiene planificada su implementación a otras empresas locales y nacionales de investigación de mercado.

\section{Formulación de hipótesis}

$H_{0}$ : Al aplicar patrones programables en una aplicación android no se podrá generar un modelo de veracidad de encuestas en zonas urbanas y rurales.

$\mathrm{H}_{1}$ : Al aplicar patrones programables en una aplicación android se podrá generar un modelo de veracidad de encuestas en zonas urbanas y rurales.

\section{Establecer un nivel de significancia:}

Nivel de significancia: $\alpha=0.05$ 


\section{Comparar:}

Tabla 2

Tabla de contingencias condición de patrones programables, según presencia de patrones de veracidad

\begin{tabular}{|c|c|c|c|c|c|c|}
\hline \multirow[t]{3}{*}{ Condición de patrones programables } & \multicolumn{4}{|c|}{ Presencia de patrones de veracidad } & \multicolumn{2}{|l|}{ Total } \\
\hline & \multicolumn{2}{|c|}{$\mathrm{Si}$} & \multicolumn{2}{|c|}{ No } & \multirow[b]{2}{*}{$n$} & \multirow[b]{2}{*}{$\%$} \\
\hline & $\mathrm{n}$ & $\%$ & $\mathrm{n}$ & $\%$ & & \\
\hline Aplicación de patrones programables & 42 & 87.5 & 6 & 12.50 & 48 & 100.00 \\
\hline No se aplican patrones programables & 48 & 100.00 & 0 & 0.00 & 48 & 100.00 \\
\hline Total & 90 & 93.75 & 6 & 6.25 & 96 & 100.00 \\
\hline
\end{tabular}

Fuente: Elaboración propia.

\section{Estadístico de prueba}

Chi Cuadrado de Homogeneidad

$X^{2}=\sum \frac{\left(f_{0}-f_{e}\right)^{2}}{f e}$

$X_{\text {comacoin }}^{2}$ rans $=\frac{n(|a d-b c|-0.5 n)^{2}}{(a+c)(b+d)(a+b)(c+d)}$

$\mathrm{X}^{2}$ calculado $=6.40$

$X^{2}$ calculado corrección de Yates $=4.44$

$X_{\text {tabla }}^{2}=3.84$

$\mathrm{X}^{2}$ calculado corrección de yates $(4.44)$ es mayor que el valor $\mathrm{X}^{2}$ tabla $=3.84$, por lo tanto, se rechaza la $\mathrm{H}_{\mathrm{o}}$.

Valor-p basada en corrección de Yates (0.035) es menor que el nivel de significancia (0.050), por lo tanto, se rechaza $\mathrm{H}_{\mathrm{o}}$.

\section{Decisión}

A un margen de error del 5\%, se concluye que al aplicar patrones programables en una aplicación android se podrá generar un modelo de veracidad de encuestas en zonas urbanas y rurales.

\section{CONCLUSIONES}

Se logró aplicar patrones programables en una aplicación android para generar un modelo de veracidad de encuestas ( $87.50 \%$ de veracidad y $12.50 \%$ de mentiras en las encuestas aplicadas), en contraste con el método tradicional que no lograba detectar la sinceridad en cuanto al diligenciamiento de las encuestas basadas en los niveles socioeconómicos, en consecuencia, se mejoró la imagen de la empresa debido al método propuesto.

Se consiguió realizar una aplicación android que permite hacer el seguimiento de los encuestadores en zonas urbanas y rurales mediante geolocalización, de esta manera la supervisión de trabajo de campo tuvo una efectividad del $100.0 \%$, reduciendo los errores no muestrales y permitiendo que la recolección de datos offline se más rápida que la recolección de datos mediante los métodos tradicionales.

Se creó una aplicación android que permite monitorear las cuotas asignadas, durante la etapa de recojo de datos en zonas urbanas y rurales, evidenciando una relación directa entre los valores estimados y parámetros poblacionales, debido al uso del PDA (dispositivos móviles personales), el mecanismo de control se realizó de manera paralela en campo mediante la implementación de reglas básicas.

\section{REFERENCIAS BIBLIOGRÁFICAS}

Albrecht, K. (2007). Intelgencia social la nueva ciencia del éxito. Zeta Bolsillo. 
Cornejo, J. (2013). Análisis, diseño e implementación de una aplicación para administrar y consultar avisos clasificados para tabletas android", Lima: Pontificia Universidad Católica del Perú.

Cottrill, C., Cámara, F., Zhao, F., Ferreira, I., Beng, H., Ben-Akiva, M., \& Zegras, C. (2013). Experiencia de la encuesta de movilidad futura en el desarrollo de una encuesta de viajes basada en teléfonos inteligentes en Singapur. Transportation Research Record, 59-67.

Gáloc, J. (2016). Diseño e implementación de un sistema de geolocalización en interiores para plataforma Android via la red Enterprise WLAN de la PUCP Título de Ingeniero Electrónico. Lima: Pontificia Universidad Católica del Perú.

García, J. (24 de Julio de 2012). Impacto de las nuevas tecnologías en el comportamiento de los consumidores. Building Talent, págs. http://www.il3.ub.edu/blog/impacto-de-las-nuevastecnologias-en-el-comportamiento-de-los-consumidores/.

INEI. (Julio de 2014). Instituto Nacional de Estadística e Informática. En Manual del Encuestador ENAHO. Lima: INEI. Recuperado el 15 de Marzo de 2017, de http://iinei.inei.gob.pe/iinei/srienaho/Descarga/DocumentosMetodologicos/2014-55/ManualEncuestador.pdf

INFODASA. (13 de Marzo de 2015). Asesores Informáticos. Recuperado el 14 de Abril de 2017, de Desarrollo de aplicaciones móviles: http://www.infodasa.com/web/text.php?id_section=171

Ku, C. (2013). Diseño e implementación de una plataforma interactiva móvil orientada al aprendizaje en temas de física . Lima: Pontifica Universidad Católica del Perú.

Kushwaha, A., \& Kushwaha, V. (March de 2011). Servicios basados en localización mediante el uso del sistema operativo Android. International Journal of Advances in Engineering \& Technology,, I, 14-20.

Mobile Marketing Association. (2011). Libro Blanco de APPS. España: SAP.

Ochoa, C. (2015). Netquest. Fundamentos de la recolección de datos online, https://cdn2.hubspot.net/hubfs/2595966/eBook-Essentials-online-data-collectionCampaign/ES_Ebook-

Esenciales\%20para\%20la\%20recolleci\%C3\%B3n\%20de\%20datos\%20online.pdf?t=1483961335

896. Obtenido de https://cdn2.hubspot.net/hubfs/2595966/eBook-Essentials-online-datacollection-Campaign/ES_Ebook-

Esenciales\%20para\%20la\%20recolleci\%C3\%B3n\%20de\%20datos\%20online.pdf?t=1483961335 896

Patil, P., Sawant, K., Desai, S., \& Shinde, A. (3 de Marzo de 2018). Task Trigger: Reminder Application based on Location. International Research Journal of Engineering and Technology (IRJET), $V$. Obtenido de www.irjet.net

PNUD. (117 de Marzo de 2015). Programa de las Naciones Unidas para el Desarrollo. Recuperado el 18 de Julio de 2015, de

http://www.pe.undp.org/content/peru/es/home/ourwork/democraticgovernance/overview.h tml

Urbano, A. (2015). Construcción de un sistema de creación y aplicación de encuestas que soporte preguntas tipo matriz de menús desplegables. Lima: Pontificia Universidad Católica del Perú. 